\title{
ON AXISYMMETRIC HEAT CONDUCTION PROBLEM FOR MULTILAYER GRADED COATED HALF-SPACE
}

\author{
Dariusz M. Perkowski, Roman Kulchytsky-Zhyhailo, Waldemar Koøodziejczyk \\ Bialystok University of Technology, Faculty of Mechanical Engineering, Bialystok, Poland \\ e-mail: perkowski.d.m@gmail.com;r.kulczycki@pb.edu.pl; w.kolodziejczyk@pb.edu.pl
}

\begin{abstract}
The technique of integral Hankel transform to find the solution of heat conduction in half-space coated by a multilayered package of homogenous laminae is applied. The half-space is heated by the given heat flux on the boundary surface. The temperature and heat flux distribution in the radial direction is analyzed for two types of coatings: 1) when the heat conductivity coefficient is described by a power or exponential function of the distance to the boundary surface; 2) multilayered coating has a periodic structure.
\end{abstract}

Keywords: temperature, heat flux, FGM, composite, homogenized model

\section{Introduction}

Modern engineering construction require the use of materials with appropriate thermal and mechanical properties which make them more and more complicated structures. To such materials belong media with functionally changing gradation properties (i.e. gradient materials). Furthermore, the surface coverage of gradient materials significantly affect the behaviour of the bodies under the influence of mechanical and thermal loads.

The formulations of the problems lead to boundary value problems of partial differential equations with varying coefficients. For the power (or exponential) law of variation of the heat conduction coefficient (or Young's modulus), the analytical methods of solutions are known (Guler and Erdogan, 2004, 2006; Liu and Wang, 2008; Matysiak et al., 2011; Kulchytsky-Zhyhailo and Bajkowski, 2015). If the thermal and mechanical properties are described by other functions, obtaining analytical solutions encounters considerable mathematical difficulties. Parallel with the application of analytical methods for the solution of partial differential equations, inhomogeneous layers are also modeled by using an approach according to which the coating is replaced by a package of homogeneous or inhomogeneous layers (Ke and Wang, 2006, 2007; Kulchytsky-Zhyhailo and Bajkowski, 2012, 2015; Liu T.-J. et al., 2008; Liu and Wang, 2009; Liu J. et al., 2011, 2012). Most studies are based on two-dimensional problems of elasticity or thermoelasticity (Barik et al., 2008; Choi and Paulino, 2008; Diao, 1999; Diao et al., 1994; Liu J. et al., 2011, 2012). However, the axisymmetric and three-dimensional problems are dealt with in a much lesser degree.

A special type of graded coating is the multilayered coating with periodic structure (Farhat et al., 1997; Vevodin et al., 2001). In the modeling of laminated half-spaces or coatings with periodic structures, it is customary to use two different approaches. In the first of these approaches the layers are considered as separate continuous media. The second approach is based on the analysis of a homogenized uniform coating whose properties are determined on the basis of the material properties and geometric characteristics of the strip of periodicity (Matysiak and Woźniak, 1987; Woźniak, 1987). The solution obtained for the laminated half-space is compared with Kulchytsky-Zhyhailo and Matysiak (2005). 
In the present work, we consider an axisymmetric problem of heat conductivity for half-space with a multilayered coating. The boundary surface is heated by the given heat flux. In parallel, we obtain: 1) analytical solutions of the problems for the coating whose heat conductivity coefficient is described by a continuous function of the distance to the boundary surface; 2) the solution for the multilayered coating with a periodic structure which is described by a homogenized uniform layer (Matysiak and Woźniak, 1987; Woźniak, 1987). We analyze the difference between the temperature and heat flux in the non-homogeneous half-space caused by the use of two different models of nonuniform coatings. The obtaining of the smallest deviations in consideration of the heat conduction problem will be a strong argument in favor for application of the proposed methods for solving axisymmetric and three-dimensional problems of thermoelasticity for the functionally graded coated half-space.

\section{Formulation of the problem}

Assume that the surface $z=h$ of the non-homogeneous half-space is heated by a heat flux $q(r)$ on the circle of radius $a$, where $r, z$ are dimensionless cylindrical coordinates referred to the linear size $a, h=H / a, H$ is thickness of the coating (Fig. 1).

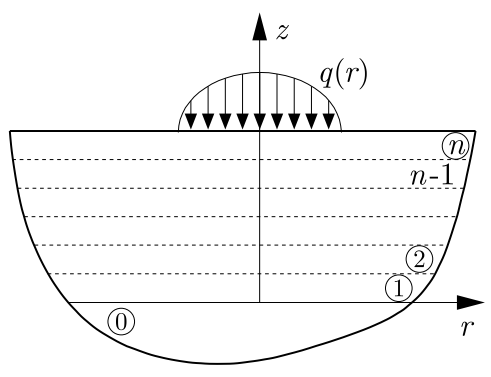

Fig. 1. The scheme of the body

The non-homogeneous half space is formed by the homogeneous half-space with the heat conductivity coefficient $K_{0}$ and a system of non-homogeneous layers with thicknesses $H_{i}$ and the heat conductivity coefficients $K_{i}, i=1,2, \ldots, n$, respectively, where the value of the parameter $n$ corresponds to the number of the layer in the package. Assume that the conditions of perfect thermal contact are realized between the layers of the coating and between the coating and the base.

The analyzed problem of theory of heat conduction is reduced to the solution of the following partial differential equations

$$
\frac{\partial^{2} T_{i}}{\partial r^{2}}+\frac{1}{r} \frac{\partial T_{i}}{\partial r}+\frac{1}{K_{i}} \frac{\partial}{\partial z}\left(K_{i} \frac{\partial T_{i}}{\partial z}\right)=0 \quad i=0,1, \ldots, n
$$

with boundary conditions imposed on the surface of the non-homogeneous half-space

$$
\frac{\partial T_{n}}{\partial z}=\frac{q(r) a}{K_{n}} H(1-r) \quad z=h
$$

conditions of perfect thermal contact between the components of the considered half-space

$$
\begin{aligned}
& T_{i}=T_{i+1} \quad K_{i}\left(h_{i}^{*}\right) \frac{\partial T_{i}}{\partial z}=K_{i+1}\left(h_{i}^{*}\right) \frac{\partial T_{i+1}}{\partial z} \\
& z=h_{i}^{*} \quad i=0, \ldots, n-1
\end{aligned}
$$

and conditions imposed at infinity

$$
T_{i} \rightarrow 0 \quad r^{2}+z^{2} \rightarrow \infty \quad i=0,1, \ldots, n
$$


where $T_{i}$ is the temperature in the $i$-th component of the non-homogenous medium, the index $i=0$ describes the parameters and functions of state in the homogeneous half-space, $h_{i}^{*}$ is the coordinate $z$ of the upper surface of the $i$-th component of the non-homogenous half-space, $h_{0}^{*}=0, h_{i}^{*}=h_{i-1}^{*}+h_{i}, h_{i}=H_{i} / a, i=1, \ldots, n, h_{n}^{*}=h, H(r)$ - Heaviside step function.

\section{Method of solution}

The solution of the boundary value problem is sought by applying the Hankel integral transformation (see Sneddon, 1966)

$$
\widetilde{T}_{i}(s, z)=\int_{0}^{\infty} T_{i}(r, z) r J_{0}(s r) d r
$$

where $J_{0}(s r)$ is the Bessel function. The solution to equation (2.1) was determined by Hankel integral transformation technique (3.1). The temperature for the homogeneous half-space in the Hankel transform space which satisfies the regularity conditions at infinity (2.4) can be written in the form

$$
\widetilde{T}_{0}(s, z)=t_{0}(s) \exp (s z)
$$

where $t_{0}(s)$ is the unknown function.

We considered the following cases.

\section{Case A}

Let $n=1$. The dependence of the heat conductivity coefficient on the coordinate $z$ is described by the formula

$$
K_{1}(z)=K_{0} \exp (\alpha z) \quad \alpha=\frac{1}{h} \ln \left(\frac{K_{S}}{K_{0}}\right) \quad 0 \leqslant z \leqslant h
$$

where $K_{S}$ is the heat conductivity coefficient on the surface of the inhomogeneous half-space. The general solution to differential equation (2.1) in the Hankel transform space specified in the coating can be written in the form

$$
\widetilde{T}_{1}(s, z)=t_{1}(s) \exp \left(\alpha^{(-)} z\right)+t_{2}(s) \exp \left(\alpha^{(+)} z\right)
$$

where $2 \alpha^{( \pm)}=-\alpha \pm \sqrt{\alpha^{2}+4 s^{2}}, t_{1}(s)$ and $t_{2}(s)$ are the unknown functions.

\section{Case A'}

Let $n=1$. The dependence of the heat conductivity coefficient on the coordinate $z$ is described by the power function

$$
\begin{aligned}
& K_{1}(z)=K^{*}(c \pm z)^{\alpha} \quad c= \pm h\left(\left(\frac{K_{S}}{K_{0}}\right)^{1 / \alpha}-1\right)^{-1} \\
& K^{*}=\frac{K_{0}}{c^{\alpha}} \quad 0 \leqslant z \leqslant h
\end{aligned}
$$

In equation (3.5) for the case $K_{0}<K_{S}$, we take sign "+", when $K_{0}>K_{S}-$ sign "-".

The Hankel transform of temperature in the coating can be written in the form

$$
\widetilde{T}_{1}(s, z)=t_{1}(s) \zeta^{p} I_{p}(s \zeta)+t_{2}(s) \zeta^{p} K_{p}(s \zeta)
$$

where $2 p+\alpha=1, \zeta=c \pm z, I_{p}(s \zeta), K_{p}(s \zeta)$ are modified Bessel functions. 
Moreover, if the analytical solution of partial differential equation with variable coefficient (2.1) is not known, the non-homogeneous coating can be replaced by a multilayered system of homogeneous layers. Their thermal properties are described by their heat conductivity coefficients

$$
K_{i}=\frac{1}{h_{i}} \int_{h_{i-1}^{*}}^{h_{i}^{*}} K_{1}(z) d z
$$

\section{Case B}

The coating composed of $n$ homogeneous layers. The general solution to equations (2.1) expressed in the Hankel transform domain takes the form

$$
\widetilde{T}_{i}=t_{2 i-1}(s) \sinh \left[s\left(h_{i}^{*}-z\right)\right]+t_{2 i}(s) \cosh \left[s\left(h_{i}^{*}-z\right)\right] \quad i=1,2, \ldots, n
$$

where $t_{i}(s), i=1, \ldots, 2 n$ are the unknown functions.

\section{Case C}

The multilayered coating with a microperiodical structure. Assume that the repeated fundamental layer comprises two homogeneous elastic sublayers with different thicknesses $\left(h_{I}\right.$ and $\left.h_{I I}\right)$ and thermal conductivities $\left(K_{I}\right.$ and $\left.K_{I I}\right)$. A large number of equations and boundary conditions on the interfaces complicates the solution of the problem. Another approach is using a homogenized model (Choi and Paulino, 2008; Diao, 1999) in which properties of the homogenized coating are determined on the base of properties of the components.

Applying the homogenized model to the coating, we solve the boundary value problem described by the equation (Matysiak and Woźniak, 1987; Woźniak, 1987)

$$
\frac{\partial^{2} T_{1}}{\partial r^{2}}+\frac{1}{r} \frac{\partial T_{1}}{\partial r}+\frac{1}{p_{1}^{2}} \frac{\partial^{2} T_{1}}{\partial z^{2}}=0
$$

where $T_{1}$ is the temperature in the homogenized coating

$$
\begin{aligned}
& p_{1}^{2}=\widetilde{K} K_{c}^{-1} \quad K_{c}=\frac{K_{I} K_{I I}}{(1-\eta) K_{I}+\eta K_{I I}} \\
& \widetilde{K}=\eta K_{I}+(1-\eta) K_{I I} \quad \eta=\frac{h_{I}}{h_{I}+h_{I I}}
\end{aligned}
$$

the boundary condition imposed on the surface of the non-homogeneous half-space

$$
\frac{\partial T_{1}}{\partial z}=\frac{q(r) a}{K_{c}} H(1-r) \quad z=h
$$

and boundary conditions of perfect thermal contact between the homogenized coating and the substrate

$$
T_{0}=T_{1} \quad K_{0} \frac{\partial T_{0}}{\partial z}=K_{c} \frac{\partial T_{1}}{\partial z} \quad z=0
$$

Boundary conditions (2.4) stay without change. The general solution to equation (3.9) in the Hankel transform takes the form

$$
\widetilde{T}_{1}=t_{1}(s) \sinh \left[(h-z) s p_{1}\right]+t_{2}(s) \cosh \left[(h-z) s p_{1}\right]
$$


Equations (3.2), (3.4), (3.6), (3.8) and (3.13) contain the unknown functions $t_{i}(s)$. These functions are obtained satisfying boundary conditions (2.2) and (2.3) (or (3.11)) and (3.12) in Case C. Satisfying the boundary conditions, the functions $t_{i}(s)$ may be written as

$$
t_{i}(s)=\frac{\widetilde{q}(s) a_{\widehat{K}}}{\widehat{t_{i}}(s)}
$$

where the functions $\widehat{t}_{i}(s)$ are obtained from the solution to linear equations (see Appendix A), $\widetilde{q}(s)$ is the Hankel transform of heat flux, $\widehat{K}=K_{n}$ in Case A, A', B and $\widehat{K}=K_{c} p_{1}$ in Case C.

Applying the inverse Hankel transform to equations (3.2), (3.4), (3.6), (3.8) and (3.13), temperature can be find at the desired location (see Sneddon, 1966)

$$
T_{i}(r, z)=\int_{0}^{\infty} s \widetilde{T}_{i}(s, z) J_{0}(s r) d s
$$

At internal points of the non-homogeneous half space $(z<h)$ the integrals are evaluated numerically using the Gaussian quadrature (Abramowitz and Stegun, 1964). On the surface $z=h$, we take into account the asymptotic behaviour of the functions $t_{2 n-1}(s)$ and $t_{2 n}(s)$ as $s \rightarrow \infty$. The continuity of the results when $z \rightarrow h$ has been also verified.

\section{Numerical results and discussion}

Assume that the heat flux is elliptically distributed as follows

$$
q(r)= \begin{cases}Q_{0} \sqrt{1-r^{2}} & \text { for } \quad r<1 \\ 0 & \text { for } r \geqslant 1\end{cases}
$$

where $r=r^{*} / a=1$ is radius of the circle heat zone and the heat flux (4.1) in the Hankel transform space takes the form (Gradshteyn and Ryzhik, 2015)

$$
\widetilde{q}(s)=\sqrt{\frac{\pi}{2}} Q_{0} \frac{J_{3 / 2}(s)}{s^{3 / 2}}
$$

where $J_{3 / 2}(s)$ is the Bessel function.

The analysis of the original relations in Case A (or A') shows that the solution of the problem of modeling of the inhomogeneous coating by the package of homogeneous layers depends on three (Case A) or four (Case A') dimensionless parameters: thickness of the coating $h$, ratio of heat conductivity coefficients on the surfaces of the non-homogeneous half space and the substrate $K_{S} / K_{0}$, parameter $\alpha$ (only for Case A') and the number of layers in the package $n$. A similar solution obtained for an inhomogeneous coating with regard to the continuous dependence of thermal properties on the coordinate is independent of the parameter $n$. In what follows, we assume that $h=0.4, K_{0} / K_{S}=2,4$, or $8, \alpha=1$ (only for Case A'), and $n=10,20,40$, or 80 .

The temperature and the heat flux in the radial direction $r$ on the considered nonhomogeneous surface are shown in Figs. 2 and 3 ((a) - Case A, (b) - Case A'). In this figures, the continuous lines correspond to the solution of the problem with continuous variation of the thermal properties. The rhombi correspond to the results obtained for the package formed by 40 homogeneous layers. The results of calculations presented in Fig. 2 show good agreement between the solutions obtained using the analyzed two models of the coating. As follows from Fig. 3, the maximum absolute error of calculation of the radial heat flux component can be observed on the end of the heated zone for $r=1$. 

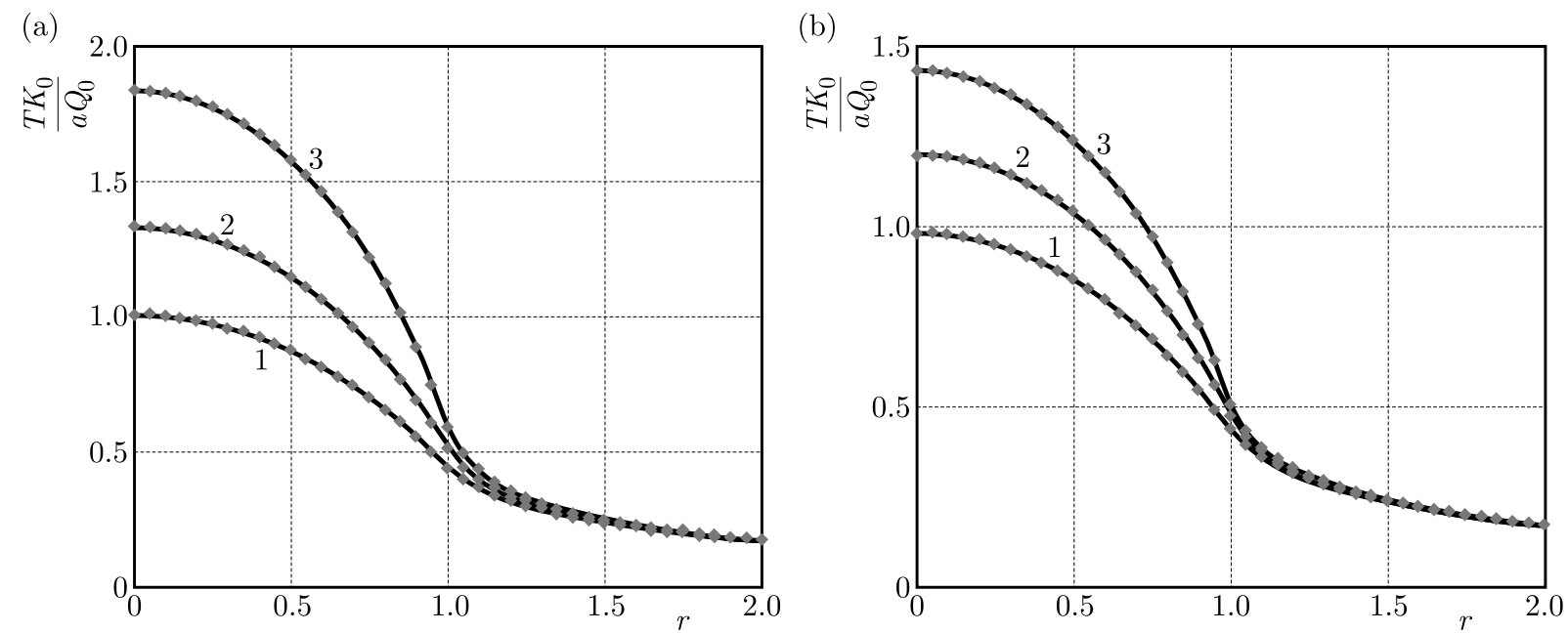

Fig. 2. The dimensionless temperature distribution on the surface $z=h$ : (a) - Case A, (b) - Case A'; $1-K_{0} / K_{S}=2,2-K_{0} / K_{S}=4,3-K_{0} / K_{S}=8 ; h=0.4$
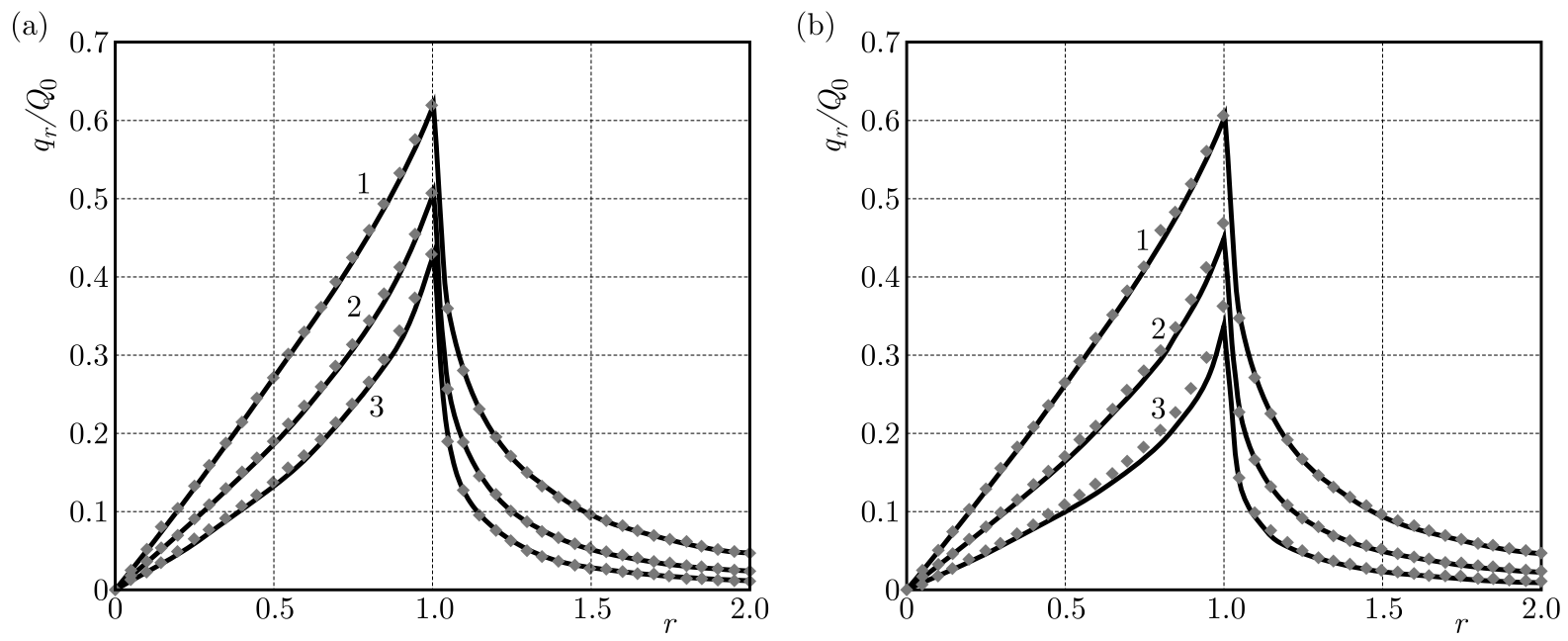

Fig. 3. The dimensionless radial heat flux on the surface $z=h$ : (a) - Case A, (b) - Case A';

$$
1-K_{0} / K_{S}=2,2-K_{0} / K_{S}=4,3-K_{0} / K_{S}=8 ; h=0.4
$$

The values of the radial heat flux in this point are presented in Table 1 . The analytical solution is presented in the rows of Table 1 with $n \rightarrow \infty$. The relative error of their evaluation with the help of modeling of the inhomogeneous coating by the package of $n$ homogeneous layers is given in columns with $n=10,20,40$, and 80 . It is easy to see that as the number of layers becomes twice larger, the corresponding error becomes almost twice lower. In the case where there are 80 layers in the package and $K_{0} / K_{S} \leqslant 8$, the error of finding the heat flux at the point $r=1, z=h$ does not exceed $2.2 \%$.

Estimating the original relations, we conclude that the distributions of temperature and heat flux in the problem of homogenized coating (Case C) depend on four dimensionless parameters: thickness of the coating $h$, ratios of heat conductivity coefficients $K_{I} / K_{0}$ and $K_{I I} / K_{0}$ and the ratio of the thicknesses of layers in the strip of periodicity $h_{I} / h_{I I}$. Similar distributions for the non-uniform coating additionally depend on the number of layers in the coating $n$. To decrease the number of input parameters, we assume that the thermal properties of one layer in the strip of periodicity coincide with the thermal properties of the base $\left(K_{I} / K_{0}=1\right.$ or $\left.K_{I I} / K_{0}=1\right)$ and the thicknesses of all layers in the stack are identical $\left(h_{I} / h_{I I}=1\right)$. We also assume that $K_{0} / K_{I}\left(\right.$ or $\left.K_{0} / K_{I I}\right)=4, h=0.2,0.4$, or 0.8 , and $n=10,20,40$, or 80 . 
Table 1. The dimensionless radial heat flux at the point $r=1, z=h(n \rightarrow \infty)$ and the errors of their evaluation as a result of modeling of the inhomogeneous coating by the package of $n$ homogeneous layers $n=10,20,40$, and 80

\begin{tabular}{|c|c|c|c|c|c|}
\hline$K_{0} / K_{S}$ & $n=10$ & $n=20$ & $n=40$ & $n=80$ & $n \rightarrow \infty$ \\
\hline \hline \multicolumn{7}{|c|}{ Case A } \\
\hline 2 & $2.79 \%$ & $1.43 \%$ & $0.68 \%$ & $0.29 \%$ & 0.6169 \\
\hline 4 & $5.40 \%$ & $2.73 \%$ & $1.29 \%$ & $0.54 \%$ & 0.5021 \\
\hline 8 & $7.79 \%$ & $3.90 \%$ & $1.82 \%$ & $0.71 \%$ & 0.4236 \\
\hline \multicolumn{7}{|c|}{ Case A' } \\
\hline 2 & $3.95 \%$ & $2.03 \%$ & $0.98 \%$ & $0.43 \%$ & 0.6022 \\
\hline 4 & $11.00 \%$ & $5.68 \%$ & $2.72 \%$ & $1.14 \%$ & 0.4562 \\
\hline 8 & $23.00 \%$ & $11.97 \%$ & $5.65 \%$ & $2.18 \%$ & 0.3435 \\
\hline
\end{tabular}

The dimensionless temperature at the centre of the heating area for different thicknesses of the coating and different numbers of layers is presented in Table 2. The temperature calculated for the homogenized model is in the last column. The relative differences between the temperature in the non-homogeneous coating and the temperature in the homogenized coating are presented in columns with $n=10,20,40$, and 80 . The upper numbers in cells have been calculated for the case $K_{0} / K_{I}=4, K_{0} / K_{I I}=1$, the lower numbers were obtained for the case $K_{0} / K_{I}=1, K_{0} / K_{I I}=4$. It can be seen that as the number of layers becomes twice larger, the errors become twice lower then. For the same value of the parameter $\delta=h / n$ (for example: $h=0.2, n=10 ; h=0.4, n=20$; and $h=0.8, n=40)$, these errors are in the same order of magnitude.

Table 2. The dimensionless temperature at the centre of the heating zone at the point $r=0$, $z=h(n \rightarrow \infty)$ and the errors of their evaluation as a result of modeling of the inhomogeneous coating by the package of $n$ homogeneous layers $n=10,20,40$, and 80

\begin{tabular}{|c|c|c|c|c|c|}
\hline$h$ & $n=10$ & $n=20$ & $n=40$ & $n=80$ & $n \rightarrow \infty$ \\
\hline \hline \multirow{2}{*}{0.2} & $1.12 \%$ & $0.56 \%$ & $0.28 \%$ & $0.14 \%$ & \multirow{2}{*}{1.0841} \\
\cline { 2 - 6 } & $-1.13 \%$ & $-0.56 \%$ & $-0.28 \%$ & $-0.14 \%$ & \\
\hline \multirow{2}{*}{0.4} & $3.35 \%$ & $1.67 \%$ & $0.83 \%$ & $0.41 \%$ & \multirow{2}{*}{1.2481} \\
\cline { 2 - 6 } & $-3.26 \%$ & $-1.64 \%$ & $-0.83 \%$ & $-0.42 \%$ & \\
\hline \multirow{2}{*}{0.8} & $7.99 \%$ & $3.92 \%$ & $1.94 \%$ & $0.96 \%$ & \multirow{2}{*}{1.3887} \\
\cline { 2 - 5 } & $-7.31 \%$ & $-3.76 \%$ & $-1.90 \%$ & $-0.96 \%$ & \\
\hline
\end{tabular}

Figures $4 \mathrm{a}$ and $4 \mathrm{~b}$ show the dimensionless radial heat flux as functions of $z$ for $r=1$ and two numbers of layers $(n=20$ and $n=40)$. In Fig. 4, the rhombi mark the numerical results obtained for the non-homogeneous laminated coating, whereas the solid lines correspond to the homogenized coating. It should be emphasized that in the case of homogenized coating, we do not know which layer of the slip of periodicity is located at the analyzed point of the coating. Hence, the radial heat flux at every point of the coating is described by the two curves. Curves 1 and 2 correspond to the heat flux acting in the layers with smaller and larger heat conductivity coefficients, respectively. In the homogeneous substrate, curves 1 and 2 coincide.

Comparing the heat flux obtained in both analyzed problems, we conclude that only in the case of the heat flux acting in the homogeneous substrate we get deviations comparable with the deviations of temperature. In the layers of the coating, the deviations of heat flux vary from $1 \%-5 \%\left(K_{0} / K_{I} \leqslant 4, n=20\right)$ up to $10 \%-20 \%$ for the heat flux acting on the boundary of the region of heating. The indicated deviations strongly depend on the gradient of the analyzed parameter in the investigated layer of the slip of periodicity, which explains the following obse- 

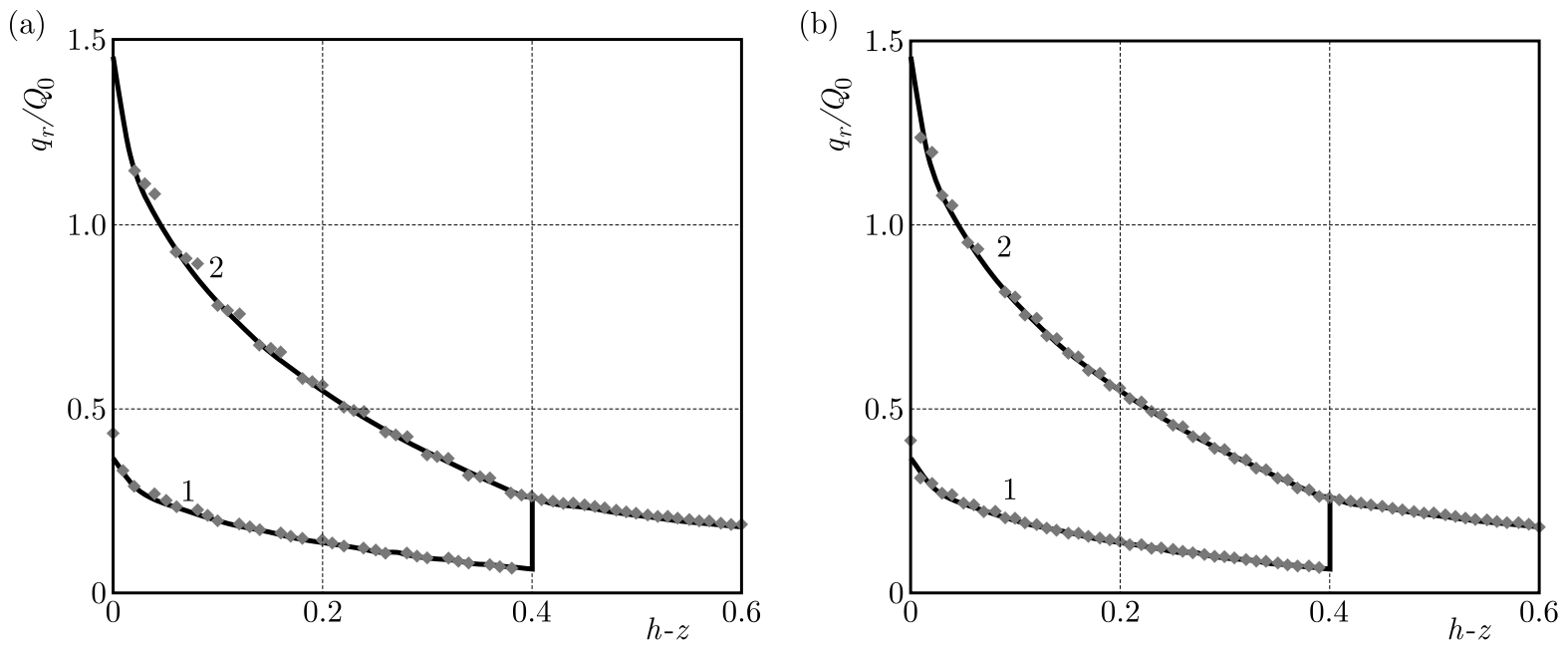

Fig. 4. The dimensionless radial heat flux on the line $r=1$ : (a) $-n=20$, (b) $-n=40 ; K_{0} / K_{I}=4$, $h=0.4, h_{I} / h_{I I}=1$

rvations: in the layers with lower heat conductivity coefficients, the deviations are much smaller (Fig. 4) and the maximum deviations are observed at the point $(1, h)$. As could be expected, the agreement between the solutions improves as the number of layers in the coating increases.

\section{Conclusions}

This paper provides the solution to the problem of the inhomogeneous half-space heated by the heat flux. It is shown that the solution to the problem for a package of 20-80 homogeneous layers is in good agreement with the analytical solution to the problem for the coating whose dependence of the heat conductivity coefficient on the coordinate $z$ is described by an exponential (or power) function. This is a strong argument for the possibility of modeling of the gradient coating with continuous variation of thermal and mechanical properties by a package of homogeneous layers.

It is shown that the solution of the axisymmetric problem of heat conductivity for the half-space with a laminated coating of the periodic structure heated by heat flux is in good agreement with the solution of the problem in which the coating is modeled by a homogenized coating. The smallest deviations are obtained while finding the temperature and heat flux in the homogeneous substrate.

\section{A. Appendix}

A system of linear equations for determination of the functions $\widehat{t}_{i}(s), i=0,1,2$ :

- Case A

$$
\begin{aligned}
& -\widehat{t}_{0}(s)+\widehat{t}_{1}(s)+\widehat{t}_{2}(s)=0 \\
& -\widehat{t}_{0}(s)+\alpha^{(-)} s^{-1} \widehat{t}_{1}(s)+\alpha^{(+)} s^{-1} \widehat{t}_{2}(s)=0 \\
& \alpha^{(-)} s^{-1} \widehat{t}_{1}(s) \exp \left(\alpha^{(-)} h\right)+\alpha^{(+)} s^{-1} \widehat{t}_{2}(s) \exp \left(\alpha^{(+)} h\right)=1
\end{aligned}
$$

- Case A'

$$
\begin{aligned}
& -\widehat{t}_{0}(s)+\widehat{t}_{1}(s) c^{p} I_{p}(s c)+\widehat{t}_{2}(s) c^{p} K_{p}(s c)=0 \\
& \widehat{t}_{0}(s) \mp \widehat{t}_{1}(s) c^{p} I_{p-1}(s c) \pm \widehat{t}_{2}(s) c^{p} K_{p-1}(s c)=0 \\
& \widehat{t}_{1}(s)(c \pm h)^{p} I_{p-1}(s(c \pm h))-\widehat{t}_{2}(s)(c \pm h)^{p} K_{p-1}(s(c \pm h))= \pm 1
\end{aligned}
$$


- Case C

$$
\begin{aligned}
& -\widehat{t}_{0}(s)+\widehat{t}_{1}(s) \sinh \left(s p_{1} h\right)+\widehat{t}_{2}(s) \cosh \left(s p_{1} h\right)=0 \\
& K_{0}\left(K_{c} p_{1}\right)^{-1} \widehat{t}_{0}(s)+\widehat{t}_{1}(s) \cosh \left(s p_{1} h\right)+\widehat{t}_{2}(s) \sinh \left(s p_{1} h\right)=0 \\
& \widehat{t}_{1}(s)=-1
\end{aligned}
$$

A system of linear equations for determination of the functions $\widehat{t}_{i}(s), i=0,1, \ldots, 2 n$ in Case B

$$
\begin{aligned}
& -\widehat{t}_{0}(s)+\widehat{t}_{1}(s) \sinh \left(s h_{1}\right)+\widehat{t}_{2}(s) \cosh \left(s h_{1}\right)=0 \\
& K_{0} K_{1}^{-1} \widehat{t}_{0}(s)+\widehat{t}_{1}(s) \cosh \left(s h_{1}\right)+\widehat{t}_{2}(s) \sinh \left(s h_{1}\right)=0 \\
& -\widehat{t}_{2 i}(s)+\widehat{t}_{2 i+1}(s) \sinh \left(s h_{i+1}\right)+\widehat{t}_{2 i+2}(s) \cosh \left(s h_{i+1}\right)=0 \\
& -K_{i} K_{i+1}^{-1} \widehat{t}_{2 i-1}(s)+\widehat{t}_{2 i+1}(s) \cosh \left(s h_{i+1}\right)+\widehat{t}_{2 i+2}(s) \sin \left(s h_{i+1}\right)=0 \quad i, \ldots, n-1 \quad i=1, \ldots, n-1 \\
& \widehat{t}_{2 n-1}(s)=-1
\end{aligned}
$$

Acknowledgments

This work was carried out within the project "selected problems of thermomechanics for materials with temperature dependent properties". The project was financed by the National Science Centre awarded based on the decision number DEC-2013/11/D/ST8/03428.

\section{References}

1. Abramowitz M., Stegun I.A., 1964, Handbook of Mathematical Functions: with Formulas, Graphs, and Mathematical Tables, United States Department of Commerce, National Bureau of Standards

2. Barik S.P., Kanoria M., Chaudhuri P.K., 2008, Steady state thermoelastic contact problem in a functionally graded material, International Journal of Engineering Science, 46, 775-789

3. Choi H.J., Paulino G.H., 2008, Thermoelastic contact mechanics for a flat punch sliding over a graded coating/substrate system with frictional heat generation, Journal of the Mechanics and Physics of Solids, 56, 4, 1673-1692

4. Dino D.F., 1999, Finite element analysis on local yield map and critical maximum contact pressure for yielding in hard coating with an interlayer under sliding contact, Tribology International, 32, $25-32$

5. Diao D.F., Kato K., Hayashi K., 1994, The maximum tensile stress on a hard coating under sliding friction, Tribology International, 27, 4, 267-272

6. Farhat Z.N., Ding Y., Northwood D.O., Aplas A.T., 1997, Nanoindentation and friction studies on Ti-based nanolaminated films, Surface and Coatings Technology, 89, 24-30

7. Gradshteyn I.S., Ryzhik I.M., 2015, Table of Integrals, Series and Products, Academic Press

8. Guler M.A., Erdogan F., 2004, Contact mechanics of graded coatings, International Journal of Solids and Structures, 41, 3865-3889

9. Guler M.A., Erdogan F., 2006, Contact mechanics of two deformable elastic solids with graded coatings, Mechanics of Materials, 38, 633-647

10. Ke L.-L., WAng Y.-S., 2006, Two-dimensional contact mechanics of functionally graded materials with arbitrary spatial variations of material properties, International Journal of Solids and Structures, 43, 18/19, 5779-5798

11. Ke L.-L., WANG Y.-S., 2007, Two-dimensional sliding frictional contact of functionally graded materials, European Journal of Mechanics - A/Solids, 26, 171-188 
12. Kulchytsky-Zhyhailo R., Bajkowski A., 2012, Analytical and numerical methods of solution of three-dimensional problem of elasticity for functionally graded coated half-space, International Journal of Mechanical Sciences, 54, 105-112

13. Kulchytsky-Zhyhailo R., Bajkowski A., 2015, Three-dimensional analytical elasticity solution for loaded functionally graded coated half-space, Mechanics Research Communications, 65, 43-50

14. Kulchytsky-Zhyhailo R., Matysiak S.J., 2005, On some heat conduction problem in a periodically two-layered body. Comparative results, International Communications in Heat and Mass Transfer, 32, 3-4, 332-340

15. LiU J., Ke L.-L., WANG Y.-S., 2011, Two-dimensional thermoelastic contact problem of functionally graded materials involving frictional heating, International Journal of Solids and Structures, 48, $18,2536-2548$

16. Liu J., Ke L.-L., Wang Y.-S., Yang J., Alam F., 2012, Thermoelastic frictional contact of functionally graded materials with arbitrarily varying properties, International Journal of Mechanical Sciences, 63, 1, 86-98

17. LiU T.-J., WANG Y.-S., 2008, Axisymmetric frictionless contact problem of a functionally graded coating with exponentially varying modulus, Acta Mechanica, 199, 151-165

18. Liu T.-J., WANG Y.-S., 2009, Reissner-Sagoci problem for functionally graded materials with arbitrary spatial variation of material properties, Mechanics Research Communications, 36, 322-329

19. LiU T.-J., WANG Y.-S., ZhAng C., 2008, Axisymmetric frictionless contact of functionally graded materials, Archive of Applied Mechanics, 78, 267-282

20. Matysiak S., Kulchytsky-Zhyhailo R., Perkowski D., 2011, Reissner-Sagoci problem for a homogeneous coating on a functionally graded half-space, Mechanics Research Communications, 38, 4, 320-325

21. Matysiak S.J., Woźniak C., 1987, On the modelling of heat conduction problem in laminated bodies, Acta Mechanica, 65, 223-238

22. Sneddon I.N., 1966 The Reissner-Sagoci problem, Proceedings of the Glasgow Mathematical Association, 7, 136-144

23. Voevodin A.A., Iarve E.V., Ragland W., Zabinski J.S., Donaldson S., 2001, Stress analyses and in-situ fracture observation of wear protective multilayer coatings in contact loading, Surface and Coatings Technology, 148, 38-45

24. Woźniak C., 1987, A nonstandard method of modelling of thermoelastic periodic composites, International Journal of Engineering Science, 25, 483-499 